\title{
Comparative Research of PCA+AdaBoost and PCA+LDA for Face Recognition Technology
}

\author{
ShaSha Fan ${ }^{1, a}$, Yang Du ${ }^{1, a}$, Jia Zhao ${ }^{1, a}$, Qiang Wang ${ }^{1, a}$, Fei Guo ${ }^{1, b^{*}}$ \\ ${ }^{1}$ School of Information Engineering, Beijing Institute of Fashion Technology, Beijing 100029, China \\ aemail:229826873@qq.com, ${ }^{b^{*}}$ email:Iunwen95@163.com
}

Keywords: Face Recognition; CA+AdaBoost; PCA+LDA

\begin{abstract}
Despite of the development of face recognition technology; face recognition rate is not high, which brings some inconvenience in our application. This paper carry on a simple introduction of PCA+AdaBoostalgorithm and PCA+LDA algorithm and makes a simple comparison at the end. PCA+AdaBoost algorithm and PCA+LDA algorithm are both relevant to the feature space, so it can be better to compare.
\end{abstract}

\section{Introduction}

The development of science and technology not only promotes the development of other industries but also promotes its own development. With the development of society, because of the production of a technological product, the corresponding requirement arises on the basis of the product. For example, face recognition technology[1] is based on camera, video camera, computer technology, and social worries about the method for identifying the identity of people, which promoted the emergence and development of face recognition technology itself. The face recognition technology is a hot technology including face image acquisition, face location, faces preprocessing, identity verification, identity search and many other links. With the continuous development of society, the functional demand for face recognition technology increases continuously and the target functions vary in various fields. The advantages of face recognition technology based on of all kinds of algorithms are different, so it is particularly important to choose a good algorithm. PCA+AdaBoost[2] and PCA+LDA[3] are two such algorithms and this paper will carry on the comparative study of the two algorithms and compare their advantages and disadvantages.

\section{The Technology of Face Recognition based on PCA+AdaBoostAlgorithm}

The algorithm based on the PCA+AdaBoost is achieved through a combination of PCA [4] and AdaBoost[5] algorithms. The following is the simple introduction of the PCA and AdaBoost.

PCA is a method for data analysis in statistics, now for the optimal representation of face images. Optimal representation is totighten the face image. The face images are achieved tightness by PCA, which any images can be through the characteristics of face of the linear weighting and approximate reconstruction of the face, weight coefficient is obtained by characteristic face project in characteristic face space, then by project to the coefficients of basis function on the low dimensional space to represent face and make face recognition.

The relevant formula of achieving face recognition by using PCA:

$\mathrm{X}=(\mathrm{X}-\overline{\mathrm{X}}) / \sqrt{\mathrm{D}}(1)$

Type $\mathrm{X}$ is the training sample image collection, the size of $\mathrm{N} * \mathrm{P}, \mathrm{N}$ is the sample, $\mathrm{P}$ is image size of, and $\mathrm{P}=$ Width * Height, $\mathrm{Y} 3$ is average image for the sample collection, $\mathrm{D}$ is variance.

$\mathrm{U}^{\mathrm{T}}\left(\mathrm{XX}^{\mathrm{T}}\right) \mathrm{U}=\wedge(2)$

In the equation, $\Lambda$ is a diagonal matrix composing by characteristic value, $U$ is a orthogonal matrix composing by characteristic value corresponding characteristic vectors, $\mathrm{X}$ is the sample image collection. Characteristic value is $\lambda \mathrm{i}(\mathrm{i}=1,2, \ldots, N), \lambda 1 \geq \lambda 2 \geq \ldots \geq \lambda \mathrm{N}$, feature vector is the Ui $(\mathrm{i}=1,2, \ldots, \mathrm{N})$. 


$$
\mathrm{W}=\mathrm{U}^{\mathrm{m}} \mathrm{X}(3)
$$

Type Um is a former $\mathrm{m}(\mathrm{m}<<\mathrm{N})$ a large eigenvalue eigenvector components, $\mathrm{W}$ is feature face that before $\mathrm{m}$.

$$
\mathrm{P}=\mathrm{WX}^{\mathrm{T}}(4)
$$

Type is a projection image collection in the feature space of X. Comparing the cosine formula and sample collection in the feature subspace projection on, can realize the face recognition.

$$
\begin{aligned}
& \mathrm{P}_{\text {test }}=\mathrm{W}\left(\mathrm{X}_{\text {test }}-\overline{\mathrm{X}}\right) \quad \mathrm{T} \\
& \mathrm{d}\left(\mathrm{P}, \mathrm{P}_{\text {test }}\right)=\frac{\mathrm{P} * \mathrm{P}_{\text {test }}}{\|\mathrm{P}\| * \mathrm{P}_{\text {test }} \|}(6)
\end{aligned}
$$

AdaBoost is an effective method for target classification bya lot of function through weighted voting mechanism.And AdaBoostmakes the simple classifier to generate strong classifier to make the error of each classifier irrelevant to each other and improve the generalizationability, the details are listed as follows:

Assuming that there are $\mathrm{M}$ simple classifiers which generate $\mathrm{M}$ strong classifiers, and those $\mathrm{M}$ strong classifiers combine to a strong classifier. The specific steps can be listed is $\chi_{\mathrm{t}}=$ $\frac{\varepsilon_{t} / T}{1-\varepsilon_{t} / T}$, weightis $\log \left(1 / \alpha_{t}\right)$, normalized weighting is $\frac{\log \left(1 / \alpha_{t}\right)}{\sum_{i=1}^{M} \log \left(1 / \alpha_{t}\right)}$, the strong classifier generated $\mathrm{h}_{\mathrm{f}}(\mathrm{x})=\arg \max _{\mathrm{y} \in \mathrm{Y}} \quad \sum_{\mathrm{i}=1}^{\mathrm{M}} \frac{\log \left(\frac{1}{\alpha_{\mathrm{t}}}\right)}{\sum_{\mathrm{i}=1}^{\mathrm{M}} \log \left(\frac{1}{\alpha_{\mathrm{t}}}\right)} \sum_{\mathrm{t}=1}^{\mathrm{T}}\left(\log \frac{1}{\beta_{\mathrm{t}}}\right) \mathrm{h}_{\mathrm{t}}(\mathrm{x}, \mathrm{y})$, while et equal to $\varepsilon \mid=$ $\frac{1}{2} \sum_{\mathrm{i}=1}^{\mathrm{N}} \mathrm{D}_{\mathrm{t}}(\mathrm{j})\left(1-\mathrm{h}_{\mathrm{t}}\left(\mathrm{x}_{\mathrm{i}}, \mathrm{y}_{\mathrm{i}}\right)+\sum_{\mathrm{y}=1}^{\mathrm{T}} \mathrm{q}_{\mathrm{t}}(\mathrm{i}, \mathrm{y}) \mathrm{h}_{\mathrm{t}}\left(\mathrm{x}_{\mathrm{i}}, \mathrm{y}\right)\right)$.

PCA+AdaBoost algorithm. Compile the PCA program (use SVD method to solve the eigenvalue and eigenvector, reduce the amount of calculation; Set separately retain 85\%, 95\%, 82\%, 92\%, 82\% energy feature vector) to produce 5 different feature subspace and generate 5 simple classifier.

Then get 5 improved strong classifiers and the weight parameters of each classifier through writing AdaBoost program (for each simple classifiers respectively using AdaBoost algorithm, and for $\mathrm{T}$ iteration) weight parameters obtained strong classifier and each classifier 5 after the lifting of the.

Different projection space corresponding to different strong classifier, and the weight of different classifier parameter $\alpha$, generating the final strong classifier.

On the test sample, the first is to project, then given by the classifier the classification results.

And then test the samples through ORL face database orYaleB face database [6]. And the face recognition rate using PCA+AdaBoost can be get. AdaBoost can greatly improve the recognition rate of PCA algorithm; therefore, it has great feasibility and practical significance to combine the two methods.

\section{Face recognition algorithm based on PCA+LDA}

Face recognition algorithm based on PCA+LDA is the combination of PCA and LDA[7] two kinds of algorithms. Through the algorithm combination, the two make up the defects of each other and make full use of the advantages of both to a certain extent. PCA algorithm has been mentioned in the above. The following is brief introduction of the LDA algorithm.

LDA is to extract the low dimensional feature with discrimination ability from high dimensional feature space, the low dimensional features can help to select a characteristic thatmake a Max ratio between samples of discrete rate between classes SB and samples in the class of discrete rate SW. The sample of discrete rate between classes SBdefinition and samples in the class of discrete rate SWdefinition as follows:

$$
\begin{aligned}
& \mathrm{S}_{\mathrm{B}}=\sum_{\mathrm{t}=1}^{\mathrm{C}} \mathrm{N}_{\mathrm{t}}\left(\mathrm{m}_{\mathrm{t}}-\mathrm{m}\right)\left(\mathrm{m}_{\mathrm{t}}-\mathrm{m}\right)^{\mathrm{T}}(7) \\
& \mathrm{S}_{\mathrm{W}}=\sum_{\mathrm{t}=1}^{\mathrm{C}} \sum_{\mathrm{x} \in \mathrm{X}_{\mathrm{t}}}\left(\mathrm{x}-\mathrm{m}_{\mathrm{i}}\right)\left(\mathrm{x}-\mathrm{m}_{\mathrm{i}}\right)
\end{aligned}
$$

In the formula, $\mathrm{C}$ is the number of categories. $\mathrm{Ni}$ is the prior probability.miis the mean value ofCi class samples.X is the sample belonging to I class.

According to the projection in the low dimensional space, samples should beseparated as far as possible.At the same time, within each category, the more intense the better. So, if $\mathrm{S}$ is a nonsingular 
matrix, the best projection direction $\mathrm{W}$ is the eigenvector of the largest ratio between samples of discrete degree between classes SB and samples in the class of discrete degree SW. The best projection functionis listed as follows:

$$
\mathrm{W}_{\text {opt }}=\arg \max \frac{\left|\mathrm{W}^{\mathrm{T}} \mathrm{S}_{\mathrm{B}} \mathrm{W}\right|}{\left|\mathrm{W}^{\mathrm{T}} \mathrm{S}_{\mathrm{W}} \mathrm{W}\right|}(9)
$$

By projecting the image onto $\mathrm{W}_{\text {opt }}$ space, we can get the global face features based on LDA algorithm.

PCA+LDA has the advantages of PCA and LDA, which makes LDA+PCA higher than LDA and PCA in face recognition rate. Here introduces PCA+LDA calculation process:

First put all centralized images as a column vector and array them in order for a data matrix;

Then calculate the feature subspace of LDAalgorithm;

Then work out feature vectorvalue and eigenvector and combine a best classifying space;

Then fuse PCA and LDA feature subspace to obtain PCA+LDA fusion feature space;

Finally, in order to get the recognition feature, Project training samples and testing samples respectively and fuse feature space. Then complete identification by using the nearest neighbor rule.

Then as same as PCA+AdaBoost algorithm, testsamples by using ORL face database or YaleB face database and Malta and face recognition rate curve can be obtained by PCA+LDA algorithm. This method can greatly compare face recognition rateof PCA+AdaBoostalgorithm and PCA+LDA algorithm by facial recognition curve. PCA+LDA algorithm not onlyachieves feature extraction of PCA algorithm but also solves small sample sets problems of LDA algorithm.

\section{Comparison of PCA+AdaBoost algorithm and PCA+LDA algorithm}

First of all, for PCA+AdaBoostalgorithm and PCA+LDA algorithm are both involved in PCA algorithm, both of them need to generate face image matrix, the feature space and the feature vector and so on. And both of themneed projection and linear weighted sum to reconstruct approximately. Its weight coefficient can be obtained by projecting face image incharacteristic face space.

PCA+LDA algorithm projects original imageonto the fusion feature space constituting by PCA+LDA algorithm, and removes a large number of redundant information of the image. At the same time, it retains the useful information of the image. With good adaptability, PCA+AdaBoost algorithm is not sensitive to light; form a strong classifier has a good feasibility and practical significance in improving the accuracy of face recognition.

The difference between PCA+AdaBoost algorithm and PCA+LDA algorithm in achieving face recognition mainly exists in AdaBoost and LDA algorithm. AdaBoost algorithm mainly gives big weight to the classification with good effect and smaller weight to the classification with poor effect.LDA algorithm is aimed at feature space,samples of discrete rate between classes SB and samples in the class of discrete rate SW. The two algorithms differ, the difference between AdaBoost algorithm and LDA algorithm led to face recognition degree difference of PCA+AdaBoost algorithm and PCA+LDA algorithm.

PCA+AdaBoost algorithm flow chart in Figure 1, and the flow chart of PCA+LDA algorithm in Figure 2. 


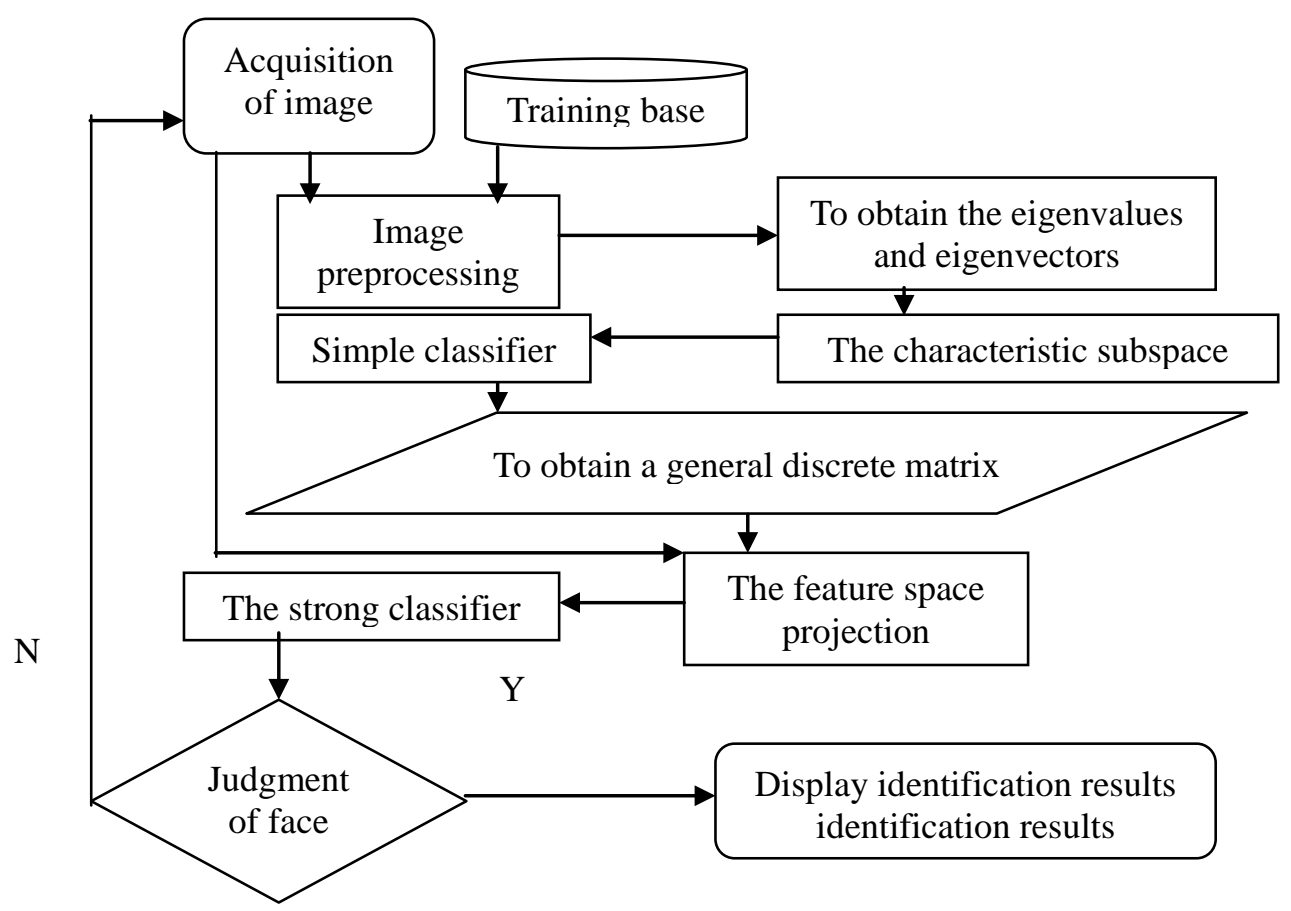

Fig. 1 Flow Chart of PCA+AdaBoostAlgorithm

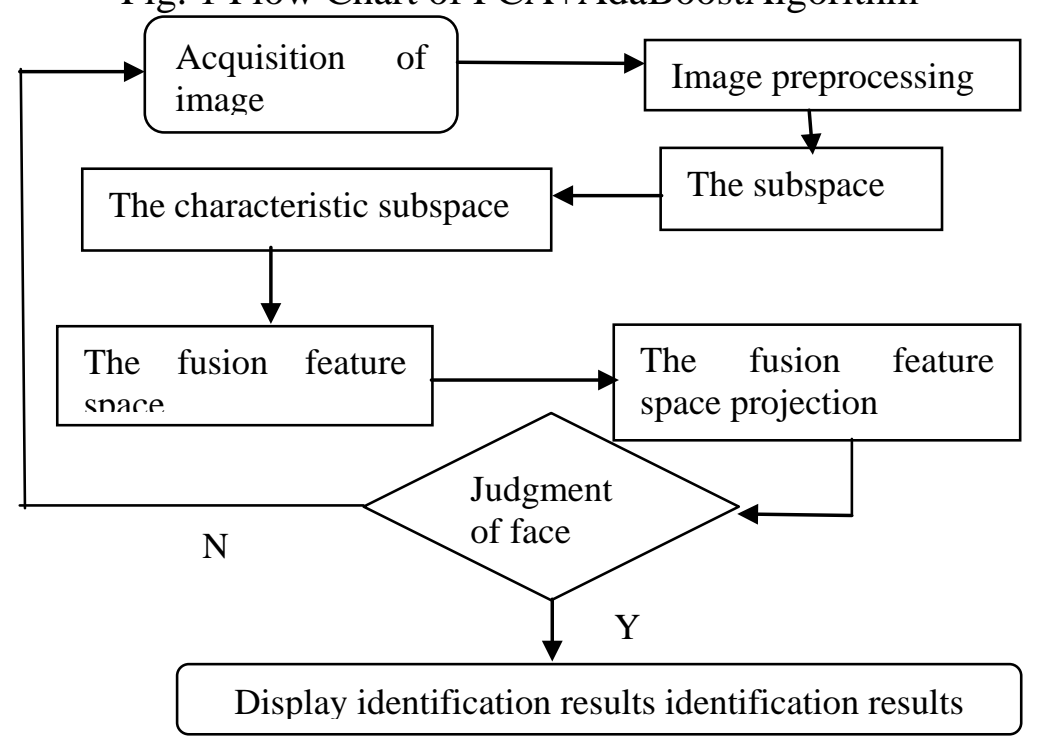

Fig. 2 Flow Chart of PCA+AdaBoostAlgorithm

\section{Experimental Analysis}

To set up a portrait gallery, the gallery contains some portraits, and named as S1, S2... and Sn, but also each onecontains a variety of different postures and expressions; in this case, respectively to simulation compare with PCA and LDA, which we combine the training feature dimension of PCA and LDA is set to 1.Now, opening a personal portrait, respectively for identification, the results are as follows:

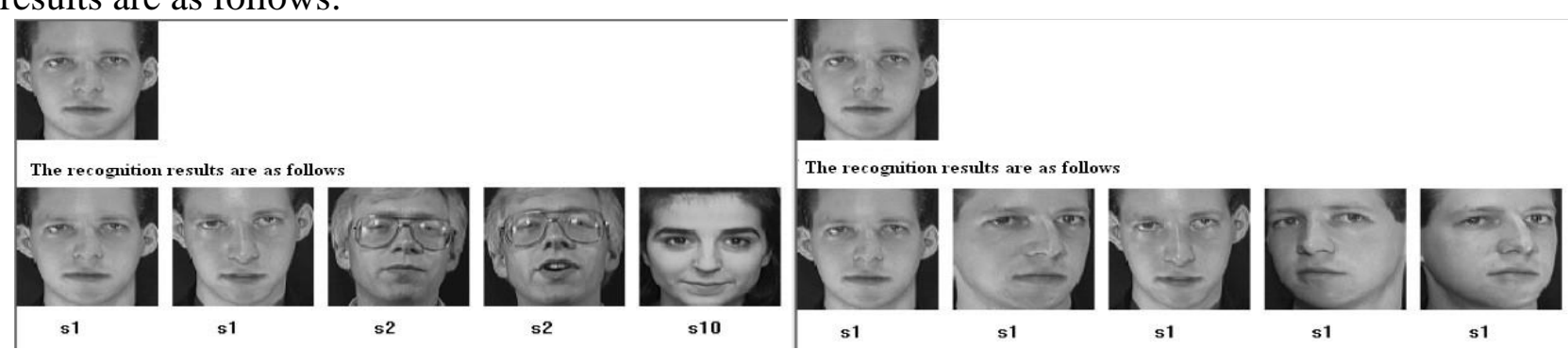

(a).PCA recognition results(b).LDArecognition results

Fig. 3Recognition Results 
From the above results it can be seen, after PCA identifying the person in full is the name for this S1 person's portrait, and after LDA recognition results are not entirely S1's portrait, which exist in error. This error is due to the advantages and disadvantages of the algorithm, the algorithm will directly affect the results. Here, we can see that compared with the LDA algorithm, PCA algorithm is more desirable, good reliability.

\section{Conclusion}

This paper briefly introduces PCA,LDA,PCA+AdaBoost and PCA+LDA algorithm and compare PCA+AdaBoost and PCA+LDA algorithm. PCA+AdaBoost and PCA+LDA algorithm have their own advantages and disadvantages, but PCA+AdaBoost algorithm is better than PCA+LDA algorithm in comparison. We believe that with the progress of science and technology, the technology aiming at the face recognition will be more mature and its function will be more powerful.

\section{Acknowledgement}

In this paper, the research was sponsored by the General Program of Science and Technology Development Project of Beijing Municipal Commission of Education (Project No.KM201410012001) and National Science and Technology Supporting Funding Project(Project No. 2013BAH41F02) andYouth InnovationFundProjectof science of Beijing Institute Of Fashion Technology(Project No.2014AL-29) and Beijing Talent Strong SchoolPlan--the InnovationTeam Project(Project No. TTBIFT_TD_002) and Beijing Key Laboratory of Digital and Interactive Media Project (Project No.KF2013-13)and 2014 College Student Research Training Program of Beijing Institute Of Fashion Technology.Teacher FeiGuo is corresponding author.

\section{References}

[1]Face recognition technology review Xue Bing, GuoXiaosong, PuPengcheng (the Second Artillery Engineering College, Xi'an 710025), Chinese Library Classification number: TP391. 4 document code: A article number: 1006- 0707 (07- 2010)0119- 03

[2]Face recognition technology based on PCA+AdaBoost algorithm South China Normal University 2010, 46 (4).

[3] Research about face recognition technology based on PCA and LDA computer science and engineering institute of Xi'an technology university Wu Wei Li Jinhui.

[4]The technology of face recognition based on modified PCA algorithm and Fisher linear discriminant analysis Shi Yuexiang, CaiZixing, Wang Xuewu, B.Benhabib, 2006 September twenty-seventh volume ninth issue Mini micro computer systems.

[5]On the AdaBoost effectiveness analysis Fu Zhongliang computer research and development ISSN1000-1239/CN11-1777/TP45 (10): 1747-17552008.

[6]Description using Multi-scale LBP feature and face recognition Wang Wei, Huang Fei, Li Jianwei, FengHailiang article number 1004 - 924X (2008) 04 - 0696 - 102008 April sixteenth volume fourth issue Optics and precision engineering.

[7] The technology of face recognition based on compressed sensing Zou Wei Li Yuanxiang, Yang Junjie, Zhou Zeming article number: 1000-3428(2012)24-0133—04 thirty-eighth volume twenty-fourth issue Vol.38 No.24. computer engineering. 